\title{
FINITE ELEMENT METHOD FOR HEAT TRANSFER PHENOMENON ON A CLOSED RECTANGULAR PLATE
}

\author{
Collins O. Akeremale \\ Department of Mathematical Sciences ${ }^{1}$ \\ Department of Mathematical Sciences \\ Federal University Lafia \\ PMB 146, Nasarawa State, Nigeria \\ ocakeremale@graduate.utm.my \\ Olusegun A Olaiju \\ Department of Mathematical Sciences \\ Department of Mathematics and Statistics \\ Federal Polytechnics Ilaro \\ PMB 50, Ogun State, Nigeria \\ oa2olaiju@graduate.utm.my \\ Yeak Su Hoe \\ Department of Mathematical Sciences ${ }^{1}$ \\ s.h.yeak@utm.my \\ ${ }^{1}$ Universiti Teknologi Malaysia \\ 81310, Skudai, Johor Bahru
}

\begin{abstract}
In the diagnosis and control of various thermal systems, the philosophy of heat fluxes, and temperatures are very crucial. Temperature as an integral property of any thermal system is understood and also, has well-developed measurement approaches. Though finite difference (FD) had been used to ascertain the distribution of temperature, however, this current article investigates the impact of finite element method (FEM) on temperature distribution in a square plate geometry to compare with finite difference approach. Most times, in industries, cold and hot fluids run through rectangular channels, even in many technical types of equipment. Hence, the distribution of temperature of the plate with different boundary conditions is studied. In this work, let's develop a finite element method (code) for the solution of a closed squared aluminum plate in a two-dimensional (2D) mixed boundary heat transfer problem at different boundary conditions. To analyze the heat conduction problems, let's solve the two smooth mixed boundary heat conduction problems using the finite element method and compare the temperature distribution of the plate obtained using the finite difference to that of the plate obtained using the finite element method. The temperature distribution of heat conduction in the 2D heated plate using a finite element method was used to justify the effectiveness of the heat conduction compared with the analytical and finite difference methods.
\end{abstract}

Keywords: finite element method, steady-state, squared plate, analytical method, closed rectangle.

DOI: $10.21303 / 2461-4262.2020 .001422$

\section{Introduction}

Heat conduction is generally simulated in two major ways, direct heat conduction problem (DHCP) estimation and inverse heat conduction problem (IHCP). In assessing the temperature distribution within conductive media, direct heat conduction simulation is commonly used. This is when the existing boundary conditions, the intensity of the heat source within or thermo-physical properties of the material body is known [1]. While the reconstruction of unknown heat flux or temperature on the surface of a body conducting heat based on temperature measurements taken at interior point or backside points is the solution of IHCP [2].

The measurement of random errors is prone to high sensitivity and as such, IHCPs are illposed mathematically. This results in astronomical disorder or perturbation in the solution. Naturally in solids, the nature of transient heat conduction is such that the perturbation on the surface penetrates and diminishes toward the interior. On the contrary, the least measurement is magnified 
at the surface, leading to large oscillation and fluctuation in the estimated surface condition when the interior point is used as an input inverse problem [2].

Temperature and heat flux of a wall in an inaccessible surface can be determined using the inverse heat conduction method by measuring the temperature on an accessible boundary. Disturbances arise in the predicted heat fluxes as a result of the noise in the measure of the temperature. To measure the temperature at two locales had been demonstrated to improve the predicted heat fluxes. Installation of an interior thermocouple can result in material inhomogeneities that change the heat flow through the wall, and in many applications, to adjust the wall to include an interior thermocouple can't be achieved. Sensitivity analysis and numerical experiments have demonstrated that the computation of the temperature can be improved by the absorbing of the measurement of heat flux at the accessible boundary. Hence, a numerical method to predict the heat transfer on an inaccessible boundary without changing the thermal boundary condition is necessary.

To establish a correct and stable estimate of the inverse solution, an appropriate approach is crucial. A major approach amongst the most efficient approaches is called future time measurements and it was first developed in a least-square sequential procedure denoted by the method of function specification by Beck [3]. The stability of the inverse problem was highly enhanced by this method. Many improvements had been proposed on the work or method of Beck [4-6]. Many authors [2, 7-13] had improved on the solution to IHCP especially with the introduction of future time measurement concepts.

Recent work [1] had used finite-difference to solve the 2D heat transfer problem and the result compared to the analytical method. The soundness of the temperature distribution was also ascertained through the finite difference scheme. However, in this work, let's intend to use a finite element for the solution of heat transfer problems and to establish or show the pattern of temperature distribution using the method of finite element. This research is important because heat transfer is not limited to regular bodies or geometry and it had been established that the finite element method is the best for irregular geometry.

Let's establish the effectiveness of temperature distribution in the heat conduction process in a $2 \mathrm{D}$ heated plate using the finite element method compared with the analytical method as reported in [1] where finite-difference was used.

\section{2D Heat Conduction in Transient}

Let's consider where an explicit method is used, the energy control to a nodal field of the size is as presented in the Fig. 1 below.

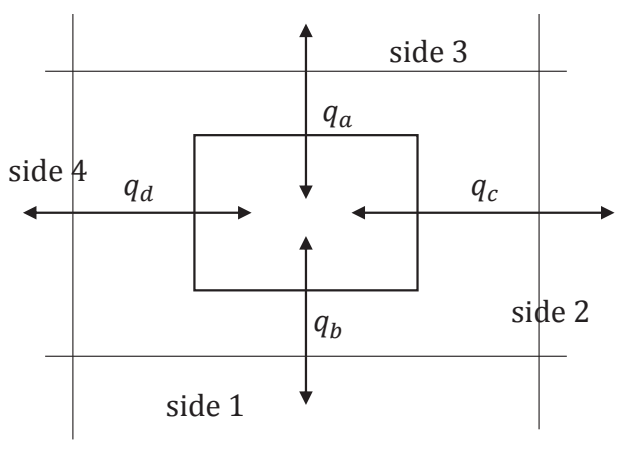

Fig. 1. Transient heat transfer phenomenon using meshes

$$
H \text { Hin }=0 \text {, }
$$

$$
\text { Hout }=H \text { in }+H \text {. }
$$

Hin=heat into the field.

$H g=$ heat generated in the field. 
Hout $=$ heat stored in the field.

Using Fig. 1 and equation (1), let's write the equation of transient heat conduction as

$$
q_{a}+q_{b}+q_{c}+q_{d}=H_{\text {out }}
$$

where

$$
q_{a}=k A_{c} \frac{\mathrm{d} T}{\mathrm{~d} y}, \quad q_{b}=k A_{c} \frac{\mathrm{d} T}{\mathrm{~d} y}, \quad q_{c}=k A_{c} \frac{\mathrm{d} T}{\mathrm{~d} y} \text { and } q_{d}=k A_{c} \frac{\mathrm{d} T}{\mathrm{~d} y}
$$

are the heat flux. $k$ is thermal conductivity, $\mathrm{d} x$ is the mesh length in the $x$ direction, $\mathrm{d} y$ is the mesh length in the $y$ direction, $A_{c}$ is the cross-sectional area of the plate. $q_{a}$ is the rate of heat flow in the side $3, q_{b}$ is the rate of heat flow in the side one $q_{c}$ is the rate of heat flow inside $2, q_{d}$ is the rate of heat flow inside 4 as Fig. 1 above.

To solve the transient phenomena in a $2 \mathrm{D}$ heat conduction plate using a finite element, the plate is discretized or divided into elements. At the boundary elements, the real boundary temperature of the plate is applied.

Since not all problems are amenable to an analytical solution, there is a need to develop an approximate method and one of the most efficient numerical methods is the finite element method which is our focus in this paper.

\section{Theoretical Formulation of Finite Difference}

Let's consider the given two-dimensional and steady heat conduction equation with zero source term and constant properties as shown below

$$
-\frac{\partial^{2} T}{\partial x^{2}}-\frac{\partial^{2} T}{\partial y^{2}}=0
$$

Using the central difference formula

$$
\frac{\partial^{2} T(x, y)}{\partial x^{2}}=\frac{T(x+h, y)+T(x-h, y)-2 T(x, y)}{h^{2}}+O(h) .
$$

Let $h=\Delta x, x=i, y=j$ such that $x+h=i+1, x-h=i-1$.

Therefore,

$$
\frac{\partial^{2} T(i, j)}{\partial x^{2}}=\frac{T(i+1, j)+T(i-1, j)-2 T(i, j)}{h^{2}}+O(h) .
$$

Similarly,

$$
\frac{\partial^{2} T(x, y)}{\partial y^{2}}=\frac{T(x, y+d)+T(x, y-d)-2 T(x, y)}{d^{2}}+O(d)
$$

Let $d=\Delta y, x=i, y=j$, such that $y+d=j+1, y-d=j-1$.

Equation (2) becomes

$$
\frac{T(i+1, j)+T(i-1, j)-2 T(i, j)}{h^{2}}+\frac{T(i, j+1)+T(i, j-1)-2 T(i, j)}{d^{2}}=f .
$$

With the same mesh length along with both $\mathrm{x}$ and y axes, i. e. $h=d$, then the approximation of the second derivatives in space using center-difference is given as 


$$
T(i+1, j)+T(i-1, j)-4 T(i, j)+T(i, j+1)+T(i, j-1)=0
$$

After applying the boundary condition, a set of algebraic equations is obtained and solved.

\section{Theoretical Formulation of Finite Element}

The finite element formulation of the steady-state heat transfer problems using the Galerkin method with constant properties is given or derived below

$$
-\frac{\partial^{2} T}{\partial x^{2}}-\frac{\partial^{2} T}{\partial y^{2}}=0 .
$$

One of the finite element methods, the Galerkin method differs from other methods in the way the basis functions are constructed. The domain $\Omega$ is portioned into disjoint subdomains called finite elements. For each element $\mathrm{K}$, the corresponding shape functions $X_{K}$ which eventually are glued together into the globally defined basis function $N_{k}$ in the Galerkin method is introduced. Galerkin approximation is different from other finite element methods (FEM) through the construction of the basis functions.

In FEM, the task is to find a linear approximate solution $T^{e^{\prime}} S$ over each element which requires the calculation of unknown $T$ values at each of the nodes of the mesh as shown in the figure below which will lead to algebraic equations as a result of many values $T$ to be determined. One of the unique properties of the finite element method in its weak form is that its solution is $C^{0}$ continuous and includes natural boundary conditions (NBCs) in its formulation.

To convert equation to weak form, let's multiply both sides of the equation with weight function say and integrate over the domain of the domain.

Let's multiply both sides of equation (2) with a test function or wave function say $w$ and integrate with the condition that the wave function is zero at the boundaries i.e $w=0$ at the boundaries

$$
\int_{\Omega}\left(w \cdot \nabla^{2} T\right) \mathrm{d} A=\int_{\Omega} f \mathrm{~d} A .
$$

Subject to

$$
\underset{\rightarrow}{\operatorname{un} . n}=u_{n}, \Gamma_{N} \text { and } T=\bar{T}, \Gamma_{D}
$$

where $T$ is the dependent or field variable

Applying Green Theorem

$$
\begin{gathered}
-\left[\int w \nabla T \cdot n \mathrm{~d} \Gamma-\int(\nabla T \cdot \nabla w) \mathrm{d} \Omega\right]-\int w \cdot f \mathrm{~d} \Omega=0, \\
-\int w \cdot u_{n} \mathrm{~d} \Gamma+\int(\nabla T . \nabla w) \mathrm{d} \Omega-\int w \cdot f \mathrm{~d} \Omega=0 .
\end{gathered}
$$

Now the Discretization by interpolation

Let

$$
w=\sum_{i} N_{i} \psi_{i}=N \psi, T=\sum_{j} N_{j} T_{j}=N T,
$$

where $N_{i}$ is the shape function at each of the nodes and $\psi$ is any constant.

Recall that $\int a \cdot b \mathrm{~d} \Omega=(a, b)$.

Therefore, $\int(\nabla T . \nabla w) \mathrm{d} \Omega=(\nabla T, \nabla w)$.

Let, $\psi=[0, \ldots 1, \ldots 0, \ldots, 0]$. 
Therefore, $w=N_{i}, i \in \Omega_{\text {in }}, i \in \partial_{N}$. Where $\Omega_{\text {in }}$ belong to internal nodes, $\partial_{N}$ belong to nodes on the Newmann boundary condition region.

Substitute (8) into (7)

$$
\int\left(\nabla \sum_{i} N_{i} \psi_{i} \cdot \nabla \sum_{j} N_{j} T_{j}\right) \mathrm{d} \Omega-\int \sum_{i} N_{i} \psi_{i} \cdot u_{n} \mathrm{~d} \Gamma-\int\left(\sum_{i} N_{i} \psi_{i} \cdot f\right) \mathrm{d} \Omega=0 .
$$

Let, $\psi=[0, \ldots 1, \ldots 0, \ldots, 0]$,

$$
\int\left(\nabla N_{i}, \nabla \sum_{j} N_{j} T_{j}\right) \mathrm{d} \Omega-\int N_{i} \cdot u_{n} \mathrm{~d} \Gamma-\int\left(N_{i} . f\right) \mathrm{d} \Omega=0
$$

where $i$ is the row of the matrix, $j$ is the column of linear system

$$
\begin{gathered}
\sum_{j}\left[\int \nabla N_{i}, \nabla N_{j}\right] T_{j} \mathrm{~d} \Omega-\int N_{i} \cdot u_{n} \mathrm{~d} \Gamma-\int\left(N_{i} . f\right) \mathrm{d} \Omega=0 \\
\sum_{j}\left[\int \nabla N_{i}, \nabla N_{j}\right] T_{j} \mathrm{~d} \Omega=\int N_{i} \cdot u_{n} \mathrm{~d} \Gamma+\int\left(N_{i} . f\right) \mathrm{d} \Omega
\end{gathered}
$$

where

$$
\begin{aligned}
& \nabla N_{j}=\left\langle\frac{\partial N_{j}}{\partial x}, \frac{\partial N_{j}}{\partial y}\right\rangle, \nabla N_{i}=\left\langle\frac{\partial N_{i}}{\partial x}, \frac{\partial N_{i}}{\partial y}\right\rangle \\
& \sum_{j}\left[\int\left\langle\frac{\partial N_{j}}{\partial x}, \frac{\partial N_{j}}{\partial y}\right\rangle \cdot\left\langle\frac{\partial N_{i}}{\partial x}, \frac{\partial N_{i}}{\partial y}\right\rangle\right] T_{j} \mathrm{~d} \Omega=\int N_{i} \cdot u_{n} \mathrm{~d} \Gamma+\int\left(N_{i} . f\right) \mathrm{d} \Omega, \\
& K_{i j}=\int_{\Omega}\left[\frac{\partial N_{i}}{\partial x} \frac{\partial N_{j}}{\partial x}+\frac{\partial N_{i}}{\partial y} \frac{\partial N_{j}}{\partial y}\right] \mathrm{d} \Omega
\end{aligned}
$$

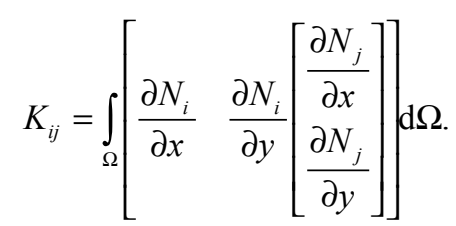

Recall that from the transformation, $N(x, y)=N(\varepsilon(x, y), \eta(x, y))$,

$$
\begin{gathered}
N_{\varepsilon}=N_{x} x_{\varepsilon}+N_{y} y_{\varepsilon}, \\
N_{\eta}=N_{x} x_{\eta}+N_{y} y_{\eta}, \\
{\left[\begin{array}{l}
N_{\varepsilon} \\
N_{\eta}
\end{array}\right]=\left[\begin{array}{ll}
x_{\varepsilon} & y_{\varepsilon} \\
x_{\eta} & y_{\eta}
\end{array}\right]\left[\begin{array}{l}
N_{x} \\
N_{y}
\end{array}\right],}
\end{gathered}
$$

where

$$
\begin{gathered}
\text { Jacobian }=\left[\begin{array}{ll}
x_{\varepsilon} & y_{\varepsilon} \\
x_{\eta} & y_{\eta}
\end{array}\right], \\
{\left[\begin{array}{c}
N_{\varepsilon} \\
N_{\eta}
\end{array}\right]=J\left[\begin{array}{l}
N_{x} \\
N_{y}
\end{array}\right],}
\end{gathered}
$$




$$
\left[\begin{array}{l}
N_{x} \\
N_{y}
\end{array}\right]=J^{-1}\left[\begin{array}{c}
N_{\varepsilon} \\
N_{\eta}
\end{array}\right]
$$

Equation (11) becomes

$$
K_{i j}=\int_{\Omega}\left[J^{-1}\left[\begin{array}{c}
N_{\varepsilon}^{i} \\
N_{\eta}^{i}
\end{array}\right]^{T} J^{-1}\left[\begin{array}{c}
N_{\varepsilon}^{j} \\
N_{\eta}^{j}
\end{array}\right]\right] \mathrm{d} \Omega .
$$

Let

$$
\begin{gathered}
B_{i}=J^{-1}\left[\begin{array}{c}
N_{\varepsilon}^{i} \\
N_{\eta}^{i}
\end{array}\right], \\
K_{i j}=\int_{\Omega}\left[B_{i}^{T} B_{j}\right] \mathrm{d} \Omega, \\
f_{i}=\int N_{i} \cdot u_{n} \mathrm{~d} \Gamma+\int\left(N_{i} \cdot f\right) \mathrm{d} \Omega .
\end{gathered}
$$

Equation (9) becomes,

$$
\begin{gathered}
\sum_{j}\left(\int_{\Omega}\left[B^{T} B\right] \mathrm{d} \Omega\right) T_{j}=\int N_{i} \cdot u_{n} \mathrm{~d} \Gamma+\int\left(N_{i} \cdot f\right) \mathrm{d} \Omega \\
{[K][T]=[F] .}
\end{gathered}
$$

This linear system of equation is then solved for each of the element and the matrices and their source terms are them assembled to form the system of equation for the whole domain.

\section{Numerical Examples}

Example 1. Let's consider a smooth 2D heat conduction plate problem in Fig. 2 below in which the temperature of the side 3 of the plate is $400 \sin (\pi x){ }^{\circ} \mathrm{C}$ and it is $0{ }^{\circ} \mathrm{C}$ on side 1 . The left and right side temperature of the plate is $0^{\circ} \mathrm{C}$ respectively with exact solution given as

$$
T_{\text {exact }}=\left(-\frac{400 e^{\pi y}}{\pi\left(e^{-\pi}-e^{\pi}\right)}+\frac{400 e^{-\pi y}}{\pi\left(e^{-\pi}-e^{\pi}\right)}\right) \sin (\pi x) .
$$

The length and width of the plate are $1 m$ as shown in the Fig. 2 below [1]

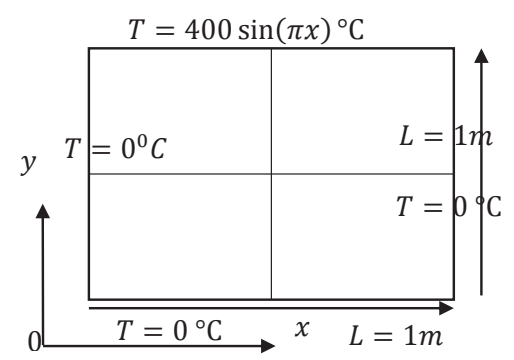

Fig. 2. 2D rectangular plate with Dirichlet condition

This problem is solved using finite element at different meshes and the temperature distributions are shown in Fig. 3, $\mathbf{4}$ below as compared with the finite difference method as shown in Fig. 10, 11. 
The FE temperature distribution is shown in Fig. 3, 4 as compared with FD temperature distribution as shown in Fig. 5, 6 below.

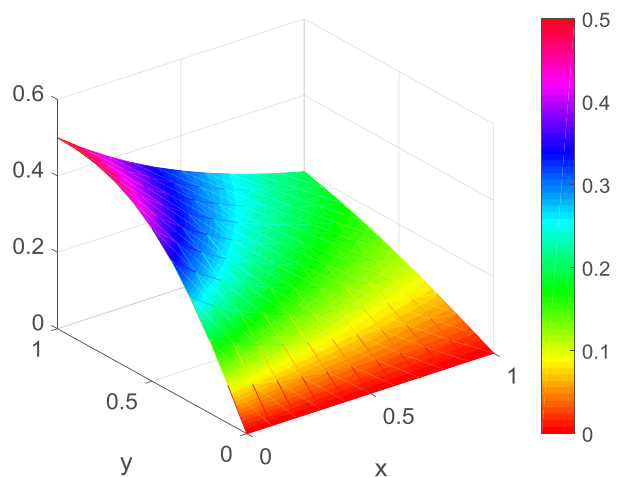

Fig. 3. Temperature distribution $(h=k=0.1)$

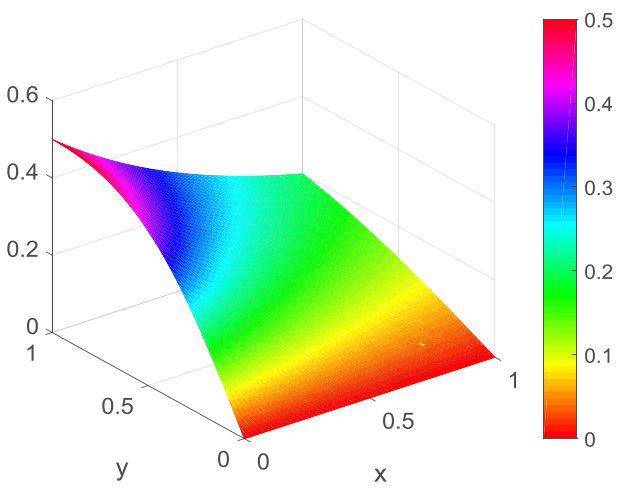

Fig. 4. FE temperature distribution for $(h=k=0.02)$

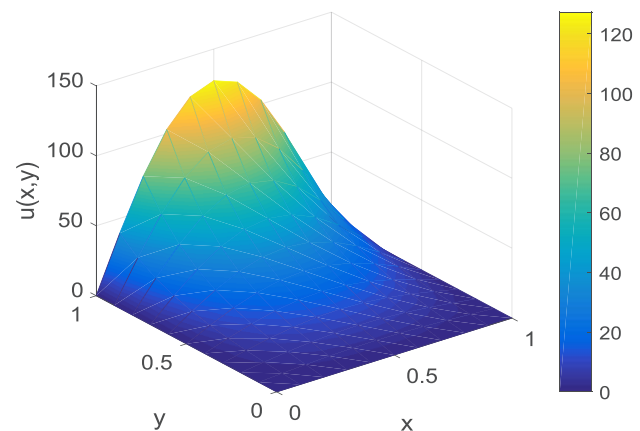

Fig. 5. Temperature distribution $(h=k=0.1)$

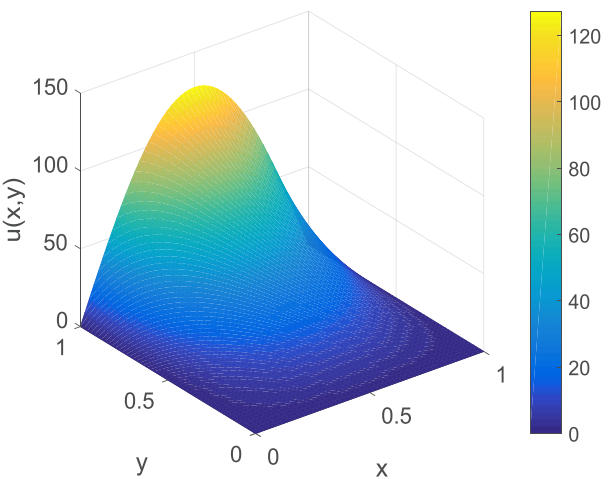

Fig. 6. FD emperature distribution for $(h=k=0.02)$ 
Fig. 3, 4 above shows that FEM produced accurate results in predicting the temperature distribution of a plate on a regular grid used in this work.

Example 2. Consider a varied boundary heat conduction plate problem in Fig. 7 below, with $\frac{1}{(1+x)^{2}+1}{ }^{\circ} \mathrm{C}$ temperature at the top boundary and bottom boundary temperature $0^{\circ} \mathrm{C}$. The left and right boundary temperatures are $\frac{y}{1+y^{2}}{ }^{\circ} \mathrm{C}$ and $\frac{y}{4+y^{2}}{ }^{\circ} \mathrm{C}$ respectively as shown in the figure below, with the exact solution given as

$$
T_{\text {exact }}=\frac{y}{\left((1+x)^{2}+y^{2}\right)}
$$

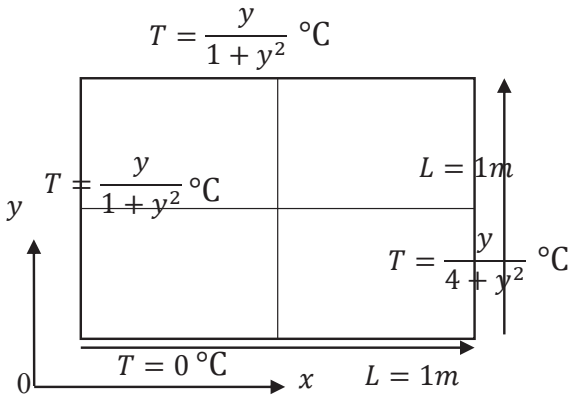

Fig. 7. 2D rectangular plate with its boundaries at different temperatures. (Dirichlet condition)

This problem is solved using finite element at different meshes and the temperature distributions are shown in Fig. 8, 9 below as compared with the finite difference method as shown in Fig. 10, 11.

The temperature distributions are as shown below.

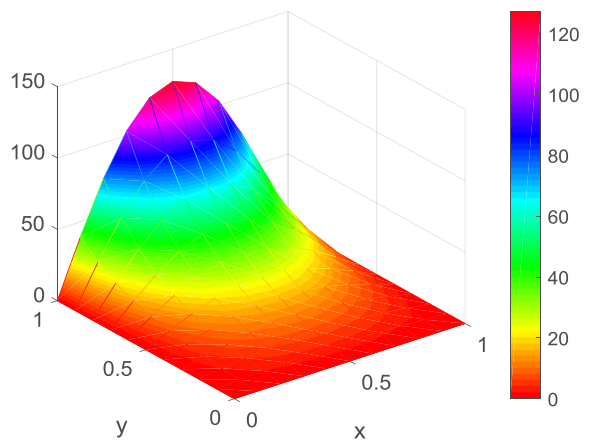

Fig. 8. FE Temperature distribution for $(h=k=0.1)$

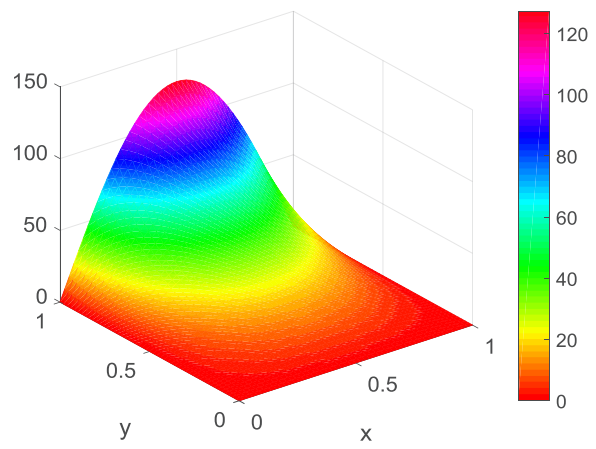

Fig. 9. FE Temperature distribution for $(h=k=0.02)$ 


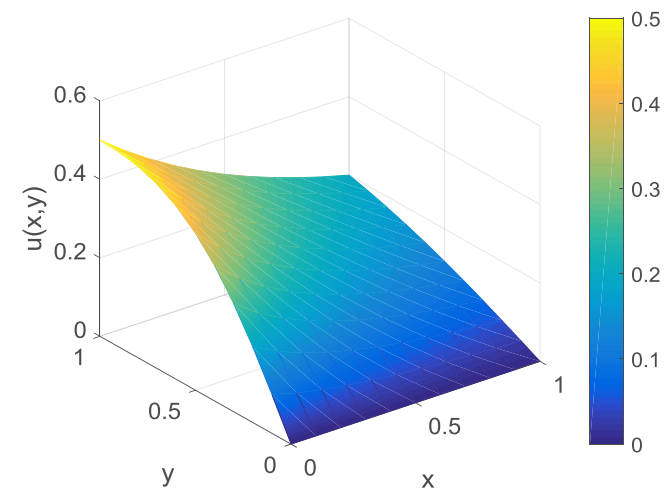

Fig. 10. FD Temperature distribution for $(h=k=0.1)$

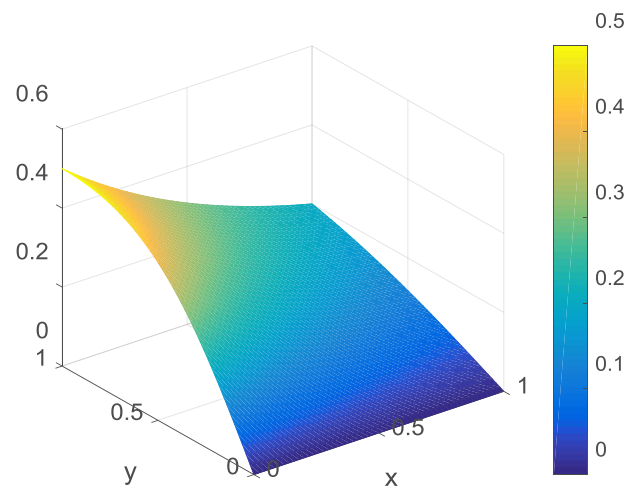

Fig. 11. FD Temperature distribution for $(h=k=0.02)$

Fig. 8-11 above show that both schemes produced accurate results in predicting the temperature distribution of a plate on a regular grid used in this work.

\section{Result and Discussion}

This section presents the results of the two examples above in terms of error using finite difference and finite element methods (Tables 1, 2).

\section{Table 1}

Numerical Error for Example 1

\begin{tabular}{ccc}
\hline & FEM & FD \\
\hline$L_{2-}$ norm error $(\mathrm{h}=0.1)$ & 0.3666543 & 1.7488 \\
$L_{2-}$ norm error $(\mathrm{h}=0.02)$ & 0.01453319 & 0.35285
\end{tabular}

Table 2

Numerical Error for Example 2

\begin{tabular}{ccc}
\hline & FEM & FD \\
\hline$L_{2-}$ norm error $(\mathrm{h}=0.1)$ & 0.0003689978 & 0.0017242 \\
$L_{2}$ norm error $(\mathrm{h}=0.02)$ & 0.00001463703 & 0.00034792
\end{tabular}

Though analytical methods are amenable to the temperature distribution of a plate with mixed boundary conditions. However, complex practical problems can't be solved analytically, hence the need for numerical methods. Though there are many numerical methods, in this work, let's adopt the finite element method and finite difference method for the solution of the heat transfer problem and compare their efficiency in terms of error. It is observed that finite element method outperform finite difference as shown in Tables 1, 2 above. The result confirms the superiority of 
finite element method compared to finite difference. Also, comparing the temperature distribution of the plate using the finite difference and finite element methods, it is observed that the changes in the mesh have no or little effect on the temperature distribution as shown in Fig. 3-6, 8-11. The distribution of temperature or heat with the mesh $h=k=0.1$ and $h=k=0.02$ are nearly the same, which shows the independency of the solution on the choice of mesh $(k$ and $h)$ both in the finite element and the finite difference methods. As shown in Fig. 3, 8 above, the distribution of temperature in the two different practical problems with $h=k=0.1$, presents a smooth $2 \mathrm{D}$ heat transfer with the heat moving from a high region of hot temperature to region low temperature while the plate cool off.

\section{Conclusion}

In conclusion, our analysis has shown that finite element method is superior to finite difference method as it gives more accurate result compared to finite difference. This explain why it has been a prefer method for engineers and scientist in addressing real life challenges. It has the ability to capture fine grid and to handle complex geometries. Finite element method is therefore recommended for high gradient problems and problems with irregular geometries.

\section{References}

[1] Deb Nath, S. K., Peyada, N. K. (2015). Numerical Study Of The Heat Transfer Phenomenon Of A Rectangular Plate Including Void, Notch Using Finite Difference Technique. International Journal of Applied Mechanics and Engineering, 20 (4), $733-756$. doi: https://doi.org/10.1515/ijame-2015-0048

[2] Gaaloul, N., Daouas, N. (2018). An extended approach of a Kalman smoothing technique applied to a transient nonlinear two-dimensional inverse heat conduction problem. International Journal of Thermal Sciences, 134, 224-241. doi: https:// doi.org/10.1016/j.ijthermalsci.2018.08.021

[3] Beck, J. V. (1970). Nonlinear estimation applied to the nonlinear inverse heat conduction problem. International Journal of Heat and Mass Transfer, 13 (4), 703-716. doi: https://doi.org/10.1016/0017-9310(70)90044-x

[4] Hensel, E., Hills, R. G. (1986). An Initial Value Approach to the Inverse Heat Conduction Problem. Journal of Heat Transfer, 108 (2), 248-256. doi: https://doi.org/10.1115/1.3246912

[5] Millan, D. N. P. (2000). Resolution of a three-dimensional unsteady inverse problem by sequential method using parameter reduction and infrared thermography measurements. Numerical Heat Transfer, Part A: Applications, 37 (6), $587-611$. doi: https://doi.org/10.1080/104077800274109

[6] Lin, S.-M., Chen, C.-K., Yang, Y.-T. (2004). A modified sequential approach for solving inverse heat conduction problems. International Journal of Heat and Mass Transfer, 47 (12-13), 2669-2680. doi: https://doi.org/10.1016/j.ijheatmasstransfer.2003.11.027

[7] Daouas, N., Radhouani, M.-S. (2004). A new approach of the kalman filter using future temperature measurements for nonlinear inverse heat conduction problems. Numerical Heat Transfer, Part B: Fundamentals, 45 (6), 565-585. doi: https://doi.org/ 10.1080/10407790490430598

[8] Wang, H.-M., Chen, T.-C., Tuan, P.-C., Den, S.-G. (2005). Adaptive-Weighting Input-Estimation Approach to Nonlinear Inverse Heat-Conduction Problems. Journal of Thermophysics and Heat Transfer, 19 (2), 209-216. doi: https://doi.org/10.2514/1.8720

[9] Daouas, N., Radhouani, M.-S. (2007). Experimental validation of an extended Kalman smoothing technique for solving nonlinear inverse heat conduction problems. Inverse Problems in Science and Engineering, 15 (7), 765-782. doi: https://doi.org/ $10.1080 / 17415970701200526$

[10] Massard, H., Orlande, H. R. B., Fudym, O. (2012). Estimation of position-dependent transient heat source with the Kalman filter. Inverse Problems in Science and Engineering, 20 (7), 1079-1099. doi: https://doi.org/10.1080/17415977.2012.712520

[11] Wan, S., Wang, G., Chen, H., Wang, K. (2017). Application of unscented Rauch-Tung-Striebel smoother to nonlinear inverse heat conduction problems. International Journal of Thermal Sciences, 112, 408-420. doi: https://doi.org/10.1016/j.ijthermalsci. 2016.11.004

[12] Wang, G., Wan, S., Chen, H., Lv, C., Zhang, D. (2017). A double decentralized fuzzy inference method for estimating the time and space-dependent thermal boundary condition. International Journal of Heat and Mass Transfer, 109, 302-311. doi: https:// doi.org/10.1016/j.ijheatmasstransfer.2017.02.001

[13] Wang, X., Wang, G., Chen, H., Zhang, L. (2017). Real-time temperature field reconstruction of boiler drum based on fuzzy adaptive Kalman filter and order reduction. International Journal of Thermal Sciences, 113, 145-153. doi: https:// doi.org/10.1016/j.ijthermalsci.2016.11.017 